\title{
Experimental factors affecting PCR-based estimates of microbial species richness and evenness
}

\author{
Anna Engelbrektson ${ }^{1}$, Victor Kunin ${ }^{1}$, Kelly C Wrighton ${ }^{2}$, Natasha Zvenigorodsky ${ }^{1}$, \\ Feng Chen ${ }^{1}$, Howard Ochman ${ }^{3}$ and Philip Hugenholtz ${ }^{1}$ \\ ${ }^{1}$ Department of Energy Joint Genome Institute, Walnut Creek, CA, USA; ${ }^{2}$ Department of Plant and Microbial \\ Biology, University of California, Berkeley, CA, USA and ${ }^{3}$ Department of Ecology and Evolutionary Biology, \\ University of Arizona, Tucson, AZ, USA
}

\begin{abstract}
Pyrosequencing of 16S rRNA gene amplicons for microbial community profiling can, for equivalent costs, yield more than two orders of magnitude more sensitivity than traditional PCR cloning and Sanger sequencing. With this increased sensitivity and the ability to analyze multiple samples in parallel, it has become possible to evaluate several technical aspects of PCR-based community structure profiling methods. We tested the effect of amplicon length and primer pair on estimates of species richness (number of species) and evenness (relative abundance of species) by assessing the potentially tractable microbial community residing in the termite hindgut. Two regions of the $16 \mathrm{~S}$ rRNA gene were sequenced from one of two common priming sites, spanning the V1-V2 or V8 regions, using amplicons ranging in length from 352 to $1443 \mathrm{bp}$. Our results show that both amplicon length and primer pair markedly influence estimates of richness and evenness. However, estimates of species evenness are consistent among different primer pairs targeting the same region. These results highlight the importance of experimental methodology when comparing diversity estimates across communities.
\end{abstract}

The ISME Journal (2010) 4, 642-647; doi:10.1038/ismej.2009.153; published online 21 January 2010

Subject Category: microbial populations and community ecology

Keywords: $16 \mathrm{~S}$ rRNA; diversity estimates; microbial community; pyrosequencing; termite

\section{Introduction}

Next-generation sequencing technologies have generated renewed interest in culture-independent $16 \mathrm{~S}$ rRNA gene-based community profiling (Tringe and Hugenholtz, 2008). 16S amplicon pyrosequencing permits a much deeper sampling of microbial communities by providing orders of magnitude more sequence information than the more traditional Sanger sequencing of PCR-clone libraries. Moreover, bar-coded 16S amplicons from multiple samples can be analyzed in parallel and provide greater sensitivity than do PCR-clone libraries (Sogin et al., 2006; Parameswaran et al., 2007). This makes it possible to examine the effects of several variables on community composition estimates, such as biases due to DNA extraction or PCR conditions.

In this study, we investigate two technical issues, namely the effects of primer choice and amplicon length on assessments of bacterial species richness and evenness. The termite P3 hindgut lumen community of Nasutitermes corniger was chosen to

Correspondence: P Hugenholtz, Microbial Ecology Program, DOE Joint Genome Institute, 2800 Mitchell Drive, Building 400, Walnut Creek, CA 94598, USA.

E-mail: phugenholtz@lbl.gov

Received 10 September 2009; revised 14 December 2009; accepted 15 December 2009; published online 21 January 2010 assess these factors because this community has been characterized extensively by PCR-clone library analysis ( $\sim 1700$ near full-length sequenced clones) and has potentially tractable diversity (Warnecke et al., 2007). As part of this investigation, we explored two hypotheses. First, we anticipated that shorter amplicons produce higher richness estimates. Given that amplicons can compete with primers for binding sites in the PCR reaction, shorter amplicons may accumulate and inhibit their own production in earlier cycles allowing rarer templates to amplify in later cycles, thereby increasing the apparent richness. In addition, we hypothesize that primer choice will have a marked effect on species evenness because of variable priming specificities and annealing kinetics (Suzuki and Giovannoni, 1996).

\section{Materials and methods}

DNA extraction

To obtain termite hindgut community DNA, the gut tracts of $25 \mathrm{~N}$. corniger worker specimens were extracted from the exoskeleton using sterile forceps. A hemi-transverse incision of the P3 hindgut compartment was made using a needle, and $2 \mu \mathrm{l}$ of $100 \mathrm{mM}$ phosphate-buffered saline was mixed with luminal contents squeezed out of 
the P3 compartment. The samples were pooled, maintained on ice, and DNA was isolated using aluminum ammonium sulfate added to cetyl trimethylammonium bromide, followed by a polyethylene glycol precipitation (Wrighton et al., 2008).

\section{PCR and 454 sequencing}

Overall, 11 amplicons ranging from 352 to 1443 nucleotides (nt) in length (Figure 1) were produced using combinations of the following broadly conserved 16S primers (it must be noted that 454 adaptor sequences and bar codes are not shown here): $27 \mathrm{~F}$ ( $5^{\prime}$-agagtttgatcMtggctcag- $3^{\prime}$ ), 357F ( $5^{\prime}$-ctcct acgggaggcagcag- $\left.3^{\prime}\right), \quad 530 \mathrm{~F}$ ( $5^{\prime}$-gtgccagcMgccgcgg- $\left.3^{\prime}\right)$, 803F (5'-attagataccctggtagtc- $\left.3^{\prime}\right), 926 \mathrm{~F}\left(5^{\prime}\right.$-aaactYaaaK gaattgacgg- $\left.3^{\prime}\right), 1114 \mathrm{~F}$ ( $5^{\prime}$-gcaacgagcgcaaccc- $\left.3^{\prime}\right), 342 \mathrm{R}$ (5'-ctgctgcSYcccgtag-3'), 519R (5'-gWattaccgcggcK gctg-3'), 787R ( $5^{\prime}$-ctaccagggtatctaat-3'), 907R ( $5^{\prime}$-ccgt caattcMtttRagttt-3), 1100R (5'-gggttgcgctcgttg- $\left.3^{\prime}\right)$ and 1392R (5'-acgggcggtgtgtRc-3').

To multiplex amplicons for inclusion on a single sequencing run, the common primer in each reaction $(27 \mathrm{~F}$ or $1392 \mathrm{R})$ was bar coded on the $5^{\prime}$ end with five unique bases between the $454 \mathrm{~A}$ adaptor sequence $\left(5^{\prime}\right.$-gcctccctcgcgccatcag- $\left.3^{\prime}\right)$ and the conserved 16S rRNA primer sequence. Rules for bar coding were established to reduce the likelihood of ambiguities due to potential homopolymeric errors; (1) barcodes cannot start with the same nt as the 454 adaptor ends; (2) bar codes cannot end with the same nt as the first nt in the $16 \mathrm{~S}$ primer; (3) there can be no more than two successive occurrences of the same nt within the bar code; and (4) each

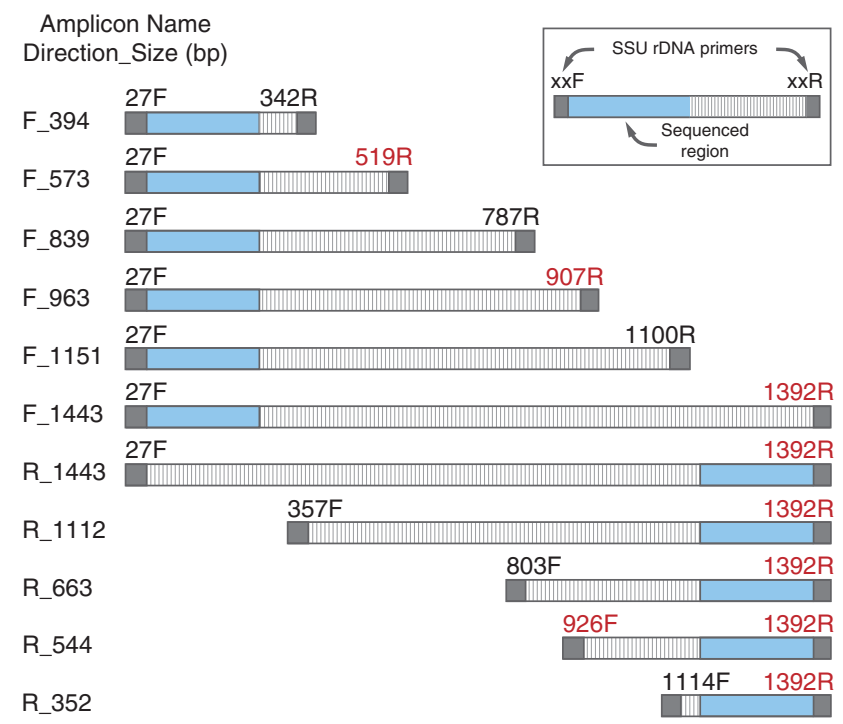

Figure 1 Experimental design showing amplified regions of the 16S rRNA gene. Amplicon names to the left of the figure denote amplicon length including primers and the orientation of sequencing, forward (F) or reverse (R). Representations of amplicons show the region sequenced (blue) and forward (F) or reverse (R) primers (gray) used to produce the amplicon. Universal primers are represented in red typeface and domain-level primers are in black. bar code must differ from other bar codes by at least two bases. The other primer in each pair was not bar coded but did incorporate the 454 B-adaptor ( $5^{\prime}$-gccttgccagcccgctca- $\left.3^{\prime}\right)$ at its $5^{\prime}$ end.

For each primer pair, PCR was performed in triplicate and pooled to minimize random PCR bias. Each $20 \mu \mathrm{l}$ reaction consisted of 0.5 Units Taq (GE Healthcare, Waukesha, WI, USA), $2 \mu \mathrm{l}$ of supplied $10 \times$ buffer, $0.4 \mu \mathrm{l}$ of $10 \mathrm{mM}$ dNTP mix (MBI Fermentas, Burlington, Ontario, Canada), $0.6 \mu \mathrm{l}$ of $10 \mathrm{mg} \mathrm{ml}^{-1}$ bovine serum albumin (New England Biolabs, Ipswich, MA, USA), $0.2 \mu \mathrm{l}$ of each $10 \mu \mathrm{M}$ primer and $10 \mathrm{ng}$ of template DNA. Each reaction proceeded under the following conditions: $95^{\circ} \mathrm{C}$ for $3 \mathrm{~min}$; 25 cycles of $95{ }^{\circ} \mathrm{C}$ for $30 \mathrm{~s}, 55^{\circ} \mathrm{C}$ for $45 \mathrm{~s}$ and $72^{\circ} \mathrm{C}$ for $90 \mathrm{~s}$; followed by a final extension at $72{ }^{\circ} \mathrm{C}$ for $10 \mathrm{~min}$. Amplification products were purified on Qiagen MinElute PCR columns (Qiagen, Valencia, CA, USA) according to the manufacturer's instructions and quantified using a Qubit fluorometer (Invitrogen, Carlsbad, CA, USA). To obtain a similar number of reads from each sample, amplicons were mixed in equal concentrations. Emulsion PCR and sequencing were performed using a GS FLX emPCR amplicon kit according to the manufacturer's protocols (454 Life Sciences, Branford, CT, USA).

\section{Informatics analysis}

Pyrosequencing flowgrams were converted to sequence reads using the standard software provided by 454 Life Sciences. Reads were processed using the computational pipeline described in the study by Kunin et al. (2010). Briefly, the reads were end trimmed with LUCY (Li and Chou, 2004) using an accuracy threshold of $0.5 \%$ per base error probability, and then the bar code and primer sequences were removed from the $5^{\prime}$ end of the read. Reads lacking exact matches to a bar code and primer were discarded. All remaining reads were uniformly truncated to $220 \mathrm{nt}$ on the basis of the length histogram of the quality-trimmed reads (not shown). Reads shorter than $220 \mathrm{nt}$ were excluded from further analyses. Identical 220-nt reads were removed and unique sequences compared by blastn using a word length of 25 . The blast output was filtered to remove all pairwise matches with similarities $<97 \%$ across the entire read length and clustered using the Markov Cluster algorithm using default parameters (van Dongen, 2000). Overall, $97 \%$ operational taxonomic units (OTUs) were classified taxonomically by blastn comparisons against the greengenes database (DeSantis et al., 2006) using a word length of 25 . Pass rates for each step of the processing pipeline were recorded.

To assess richness, rarefaction curves and bootstrapping were performed using an in-house script that plots randomly sampled clustered reads as a function of the number of $97 \%$ OTUs. To assess evenness, rank abundance curves were prepared using $97 \%$ OTUs with $\geqslant 0.5 \%$ relative abundance, 
averaged for each region. Simpson's measure of evenness $\left(E_{1 / D} ; D=\sum(n / N)^{2}\right.$, where $n$ is the number of organisms for a given species and $N$ the number of organisms for all species) was calculated for each amplicon using the statistical program R (RDC Team, 2008). This metric is insensitive to the taxa richness and ranges from 0 to 1 , with 0 representing complete dominance and 1 representing an evenly structured community. We statistically compared differences in evenness estimates between the primer regions using a two-sample $t$-test (Minitab Inc., State College, PA, USA). For the F_573 amplicon with anomalous OTU evenness (see Supplementary Figure S2), we estimated the presence of a mismatch in the 519R primer for each OTU on the basis of the closest matched fulllength greengenes sequence. Relative abundances of phyla were calculated using the greengenes classifications of the OTUs. Analysis of Similarity (ANOSIM) in the statistical package Primer V (Plymouth Marine Laboratory, Plymouth, UK), an analog to the standard univariate one-way ANOVA (analysis of variance) designed for ecological data, was performed on phylum-level pyrosequencing data to statistically assess assemblage differences between primer pairs (Clarke 1993). For statistical testing, we applied either an ANOSIM or two-sample $t$-test, and considered a probability ( $P$-value) less than 0.05 to denote significance.

\section{Data submission}

454 GS FLX flowgrams (sff files) were submitted to the Short Read Archive database at NCBI (accession no. SRA009438).

\section{Results and Discussion}

To determine the effect of primer pair and amplicon length on 16S rRNA-based community composition estimates, we assessed OTU richness (number of OTUs) and evenness (relative abundance of OTUs) for a range of amplicons obtained from a termite hindgut community using bar-coded pyrosequencing (Sogin et al., 2006; Parameswaran et al., 2007). 16S rRNA genes were PCR amplified using a combination of broad-specificity (domain or universal) primers with 454 FLX adaptor sequences. A total of 11 amplicons, ranging in size from 352 to $1443 \mathrm{bp}$, were prepared with primer sets that spanned either the V1 and V2 (sequenced forward from $27 \mathrm{~F}$ ) or the $\mathrm{V} 8$ regions (sequenced reverse from 1392R) of the 16S rRNA gene (Figure 1). The technical replicates of four amplicons (namely F_394, F_573, R_352 and R_544; Figure 1) were used to compare differences between amplicon data sets with the variation inherent in the method.

\section{Amplicon pyrosequencing data processing and statistics}

A total of 680744 pyrosequence reads (termed 'pyrotags') were produced from the amplicons
(Supplementary Table S1). Quality-based trimming resulted in the loss of $18.6 \%$ of data, and a further $17.6 \%$ of data were lost because of short sequence length or no identifiable bar code. In total, $36.2 \%$ of reads were excluded after these quality-filtering steps (Supplementary Table S1); however, this loss was not uniform as longer amplicons contributed disproportionately to those eliminated (Supplementary Figure S1). Amplicons $>1 \mathrm{~kb}$ in length had failure rates $>90 \%$ and were not included in subsequent analyses. However, it is worth noting that amplicons as long as $963 \mathrm{bp}$ had pass rates $>50 \%$ (Supplementary Table S1), despite recommendations by manufacturers to limit amplicons to $<500$ bp.

Sequences that passed quality filtering were trimmed to a uniform length of 220 bases to facilitate comparative analyses. Trimmed reads were grouped into clusters with a 97\% identity threshold producing a total of 2269 OTUs of which 1617 and 652 were from the forward (V1-V2) and reverse (V8) regions, respectively. The applied quality-filtering and clustering parameters were previously shown to minimize the effect of pyrosequencing errors on microbial diversity estimates (Kunin et al., 2010).

\section{Species richness}

The first hypothesis was that, for a given number of reads, shorter 16S rRNA gene amplicons yield greater species richness than do longer amplicons. To estimate species richness, rarefaction curves were generated by randomly sampling reads and plotting the number of novel $97 \%$ OTUs against the number of reads sampled (Figure 2). Most

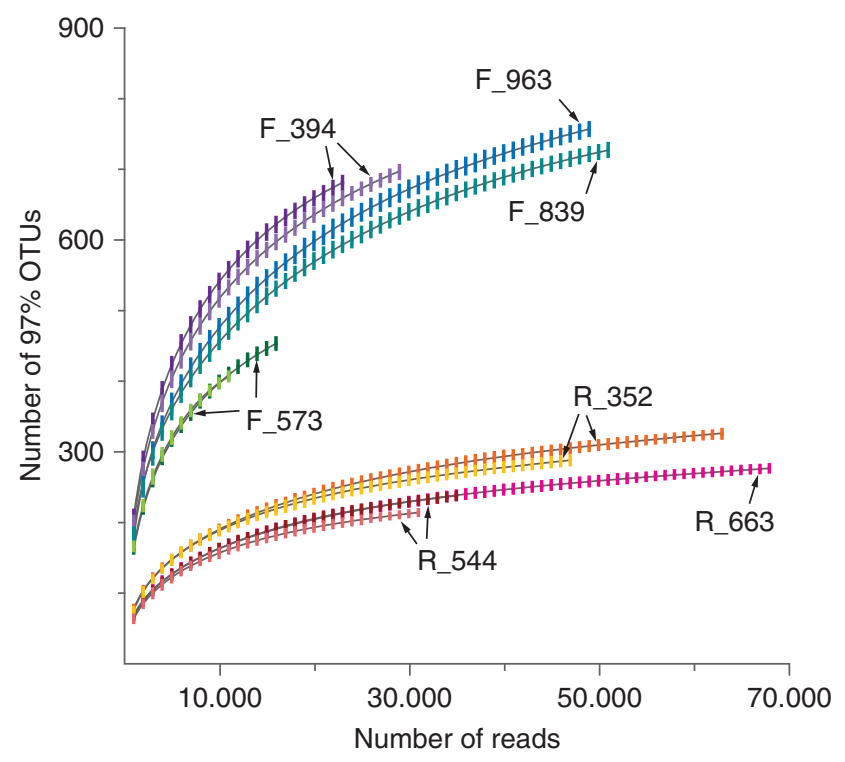

Figure 2 Rarefaction curves of the 97\% OTUs for different length amplicons from forward (V1 and V2) and reverse (V8) regions of the 16S rRNA molecule. Colored hatching represent 95\% confidence intervals. 
noticeably, forward amplicons all produced markedly (approximately threefold) higher OTU richness estimates than did reverse amplicons (Figure 2). This is due to higher sequence variability in the $\mathrm{V} 1-\mathrm{V} 2$ region than the reverse V8 region as has recently been observed by Youssef et al. (2009). Therefore, OTU richness estimates provided by $16 \mathrm{~S}$ pyrotags can vary according to the particular region surveyed, and absolute richness estimates based on different portions of the 16S rRNA gene should not be compared directly.

Within-region comparisons showed that the shortest amplicons produced higher richness estimates than did longer amplicons (Figure 2), although this trend does not seem to hold beyond 400-bp fragments (for example, F_963 seems to indicate higher richness than does F_839). The apparently much lower richness estimate of F_573 compared with other forward amplicons is likely due to a mispriming effect biasing against many phylotypes (see below). It should also be noted that technical replicates of some of the shorter amplicons resulted in different richness estimates, suggesting that rare populations were not reproducibly sampled despite the relative simplicity of the community studied. A similar relationship between amplicon length and estimated richness was observed by Huber et al. (2009) using 16S rRNA gene clone library data from two hydrothermal vent fluid samples. In this case, 100-bp amplicons produced significantly higher estimates of richness than did 400- or 1000-bp products.

\section{Species evenness}

Our second hypothesis posited that primer choice affects the relative abundance of OTUs (that is, species evenness). Whereas many primers designed to amplify $16 \mathrm{~S}$ rRNA genes are broadly conserved, no primer pair is truly universal because of basepairing exceptions present in one or more lineages targeted by broadly conserved primers (Hugenholtz and Goebel, 2001). However, the extent of this problem on PCR-based community profiling has not been systematically addressed.

Rank abundance curves of the dominant OTUs ( $>0.5 \%$ relative abundance in at least one primer set) were prepared by averaging OTU abundances across amplicons (excluding F_573, see below). This was performed separately for each region, as forward and reverse data are not directly comparable. The relative abundance of the dominant reverse OTUs was greater than the dominant forward OTUs (Figure 3), because of higher sequence conservation in the V8 region (Youssef et al., 2009) resulting in larger clusters of reverse reads at the $97 \%$ identity threshold than forward reads. To corroborate our interpretation of the curves, we calculated Simpson's inverse index of diversity $\left(E_{1 / D}\right)$ for each amplicon (Supplementary Table S2). A two-sample $t$-test performed on $E_{1 / D}$ values for each amplicon

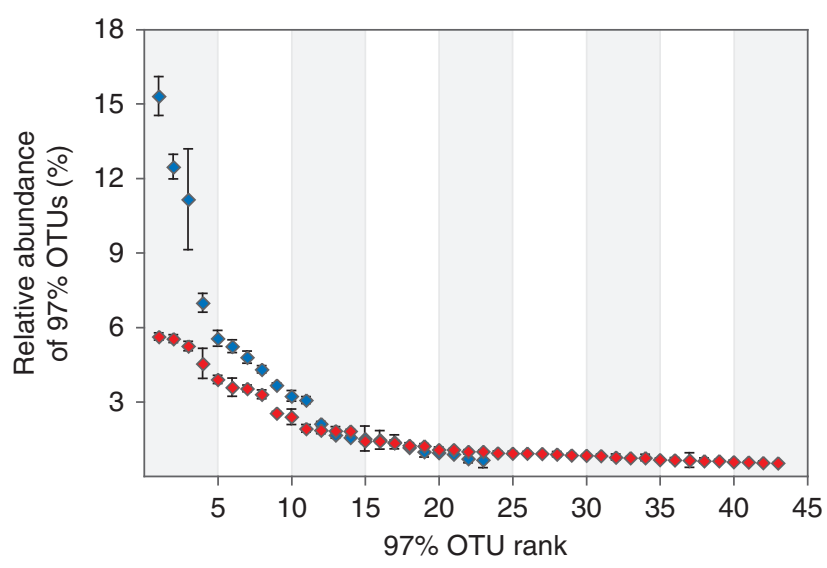

Figure 3 Rank abundance curves of the top 97\% OTUs in the forward V1-V2 (red diamonds) amplicons and reverse V8 (blue diamonds) amplicons. Standard errors are shown.

confirmed that the 16S rRNA regions (V1-V2 vs V8) evaluated in this study resulted in statistically different estimates of evenness $(P<0.05 ; P=0.012)$.

Remarkably, all of the primer pairs within either the forward or the reverse region (with the exception of $\left.F \_573\right)$ produced very similar estimates of species evenness for the dominant OTUs, as evidenced by the low standard error for most OTUs (Figure 3). The single outlier to this trend, amplicon F_573, produced markedly different OTU abundances (Supplementary Figure S2) attributable to a C:A mismatch between the 519R primer and 16S rRNA gene templates at Escherichia coli position 534, three bases from the $5^{\prime}$ end of the primer sequence. Such mismatches are customarily considered as having little or no impact on PCR because extension occurs from the $3^{\prime}$ end (Bru et al., 2008). However, the addition of the 18-bp $454 \mathrm{~B}$ adaptor to the $5^{\prime}$ end seems to have sufficiently destabilized the binding of this primer to mismatched templates, thereby favoring the amplification of perfectly matched templates. This resulted in a consistent overrepresentation of perfectly matched (T:A) templates coupled with an underrepresentation of C:A mismatched templates (Supplementary Figure S2). Therefore, primer selection can significantly affect species evenness if base variations in templates are not accounted for by degeneracies in the primer sequence. However, when these variations are addressed, evenness of dominant OTUs is highly reproducible between different primer pairs targeting the same region.

To compare the phylogenetic diversity uncovered in pyrosequence data from each region to previous estimates of the community structure in the termite hindgut, we classified all OTUs by blastn against the greengenes database (DeSantis et al., 2006) and then amalgamated the OTUs at phylum level (Figure 4). With the exception of F_573, estimates of the Nasutitermes hindgut community structure from the forward and reverse amplicons were not significantly different $(P>0.05 ; P=0.10, R=0.556)$, 
646

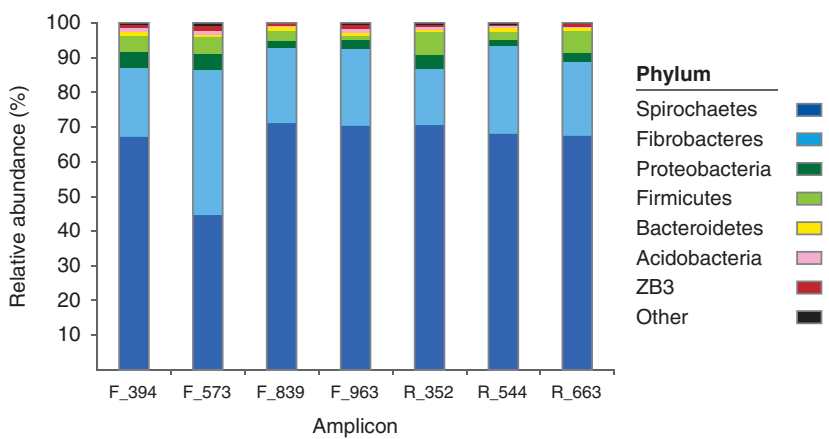

Figure 4 Relative abundance of bacterial phyla in the termite hindgut for each amplicon. Data from each technical replicate pair were averaged.

despite the difference in OTU granularity between regions (Figure 3). The dominant phylum, the Spirochaetes, comprises $67-71 \%$ of the reads in each amplicon data set, followed by the Fibrobacteres $(16-25 \%)$ and a handful of other phyla each representing $>1 \%$ of reads, including Proteobacteria, Firmicutes, Bacteroidetes, Acidobacteria and candidate phylum ZB3 (Figure 4). These results are consistent with previous PCR-based (Warnecke et al., 2007) and fluorescence in situ hybridizationbased (Hongoh et al., 2006) profiles of Nasutitermes spp. hindgut communities. In contrast, Spirochaetes were significantly underrepresented and Fibrobacteres significantly overrepresented in the $\mathrm{F} \_573$ sample because of the aforementioned C:A mismatch in most Spirochaetes and T:A match in most Fibrobacteres.

\section{Conclusions}

This study tested the hypotheses that shorter pyrotag amplicons produce higher richness estimates and that primer choice affects species evenness. Our results show that the shortest amplicons tested ( $<400 \mathrm{bp}$ ) produce higher richness estimates than do longer amplicons. However, regional variation in the $16 \mathrm{~S}$ rRNA molecule has a much greater effect on apparent richness. Within a common region, primer choice had little effect on evenness of dominant OTUs ( $>0.5 \%$ abundance), provided that template mismatches are accommodated for by degeneracies in the primer. This surprising reproducibility may have been facilitated by the use of a common primer. However, pronounced differences in evenness were observed between the two regions of 16S rRNA tested because of differences in sequence conservation. Despite the observed inconsistencies in both richness and evenness estimates between variable regions, the inferred community structure at higher taxonomic ranks (phylum) was consistent between amplicons and regions and to previous estimates of community structure from the termite hindgut. We conclude that species $(97 \%$ OTUs) evenness and richness should not be directly compared between different regions of the $16 \mathrm{~S}$
rRNA molecule. However, species evenness estimated using different primer pairs targeting the same region may be reliably compared.

\section{Acknowledgements}

We thank Rudolf Scheffrahn and Falk Warnecke for providing termites and Rebecca Daly for discussions about statistical analyses. VK was supported in part by NSF Grant no. OPP0632359 and KW by a Chang-Lin Tien Scholarship in Environmental Sciences and Biodiversity. The study was also supported by a grant from the Simon Family Fund and was performed under the auspices of the US Department of Energy's Office of Science, Biological and Environmental Research Program, as well as by the University of California, Lawrence Berkeley National Laboratory under contract no. DE-AC02-05CH11231, Lawrence Livermore National Laboratory under contract no. DE-AC52-07NA27344 and Los Alamos National Laboratory under contract no. DE-AC02-06NA25396. In memory of FWN Hugenholtz (1924-2009).

\section{References}

Bru D, Martin-Laurent F, Philippot L. (2008). Quantification of the detrimental effect of a single primertemplate mismatch by real-time PCR using the 16S rRNA gene as an example. Appl Environ Microbiol 74: 1660-1663.

Clarke KR. (1993). Non-parametric multivariate analyses of changes in community structure. Aust J Ecol 18: 117-143.

DeSantis TZ, Hugenholtz P, Larsen N, Rojas M, Brodie EL, Keller $\mathrm{K}$ et al. (2006). Greengenes, a chimera-checked 16S rRNA gene database and workbench compatible with ARB. Appl Environ Microbiol 72: 5069-5072.

Hongoh Y, Deevong P, Hattori S, Inoue T, Noda S, Noparatnaraporn $\mathrm{N}$ et al. (2006). Phylogenetic diversity, localization, and cell morphologies of members of the candidate phylum TG3 and a subphylum in the phylum Fibrobacteres, recently discovered bacterial groups dominant in termite guts. Appl Environ Microbiol 72: 6780-6788.

Huber JA, Morrison HG, Huse SM, Neal PR, Sogin ML, Mark Welch DB. (2009). Effect of PCR amplicon size on assessments of clone library microbial diversity and community structure. Environ Microbiol 11: 1292-1302.

Hugenholtz P, Goebel BM. (2001). The polymerase chain reaction as a tool to investigate microbial diversity in environmental samples. In: Rochelle PA (ed). Environmental Molecular Microbiology: Protocols and Applications. Horizon Scientific Press, Norfolk, England.

Kunin V, Engelbrektson A, Ochman H, Hugenholtz P. (2010). Wrinkles in the rare biosphere: pyrosequencing errors lead to artificial inflation of diversity estimates. Environ Microbiol 12: 118-123.

Li S, Chou HH. (2004). LUCY2: an interactive DNA sequence quality trimming and vector removal tool. Bioinformatics 20: 2865-2866.

Parameswaran P, Jalili R, Tao L, Shokralla S, Gharizadeh B, Ronaghi $\mathrm{M}$ et al. (2007). A pyrosequencing-tailored nucleotide barcode design unveils opportunities for 
large-scale sample multiplexing. Nucleic Acids Res 35: e130.

RDC Team. (2008). $R$ : A Language and Environment for Statistical Computing. R Foundation for Statistical Computing: Vienna, Austria.

Sogin ML, Morrison HG, Huber JA, Mark Welch D, Huse SM, Neal PR et al. (2006). Microbial diversity in the deep sea and the underexplored 'rare biosphere'. Proc Natl Acad Sci USA 103: 12115-12120.

Suzuki MT, Giovannoni SJ. (1996). Bias caused by template annealing in the amplification of mixtures of $16 \mathrm{~S}$ rRNA genes by PCR. Appl Environ Microbiol 62: 625-630.

Tringe SG, Hugenholtz P. (2008). A renaissance for the pioneering $16 \mathrm{~S}$ rRNA gene. Curr Opin Microbiol 11: $442-446$. van Dongen SM. (2000). PhD thesis. University of Utrecht: Utrecht, http://www.micans.org/mcl/.

Warnecke F, Luginbuhl P, Ivanova N, Ghassemian M, Richardson TH, Stege JT et al. (2007). Metagenomic and functional analysis of hindgut microbiota of a wood-feeding higher termite. Nature 450: 560-565.

Wrighton KC, Agbo P, Warnecke F, Weber KA, Brodie EL, DeSantis TZ et al. (2008). A novel ecological role of the Firmicutes identified in thermophilic microbial fuel cells. ISME J 2: 1146-1156.

Youssef N, Sheik CS, Krumholz LR, Najar FZ, Roe BA, Elshahed MS. (2009). Comparison of species richness estimates obtained using nearly complete fragments and simulated pyrosequencing-generated fragments in $16 \mathrm{~S}$ rRNA gene-based environmental surveys. Appl Environ Microbiol 75: 5227-5236. 